\title{
Rapid Profiling of Chemical Constituents in Qingfei Paidu Granules Using High Performance Liquid Chromatography Coupled with Q Exactive Mass Spectrometry
}

\author{
Shuai $\mathrm{Fu}^{1} \cdot$ Rongrong Cheng ${ }^{1} \cdot$ Zilei Xiang $^{1} \cdot$ Zixin Deng $^{1,2} \cdot$ Tiangang Liu $^{1,2,3}$
}

Received: 16 June 2021 / Revised: 5 August 2021 / Accepted: 23 August 2021 / Published online: 12 September 2021

(c) The Author(s), under exclusive licence to Springer-Verlag GmbH Germany, part of Springer Nature 2021

\begin{abstract}
Qingfei Paidu (QFPD) granules have played a critical role during the Coronavirus Disease 2019 (COVID-19) in China. However, worldwide acceptance has been a problem because of the complex ingredients and unique theory of treatment. In this study, high-performance liquid chromatography (HPLC)-Q Exactive Orbitrap-mass spectrometry (MS) and the Orbitrap traditional Chinese medicine library (OTCML) were used to investigate the chemical constituents of QFPD granules. By comparing retention times, masses, isotope ion patterns, and $\mathrm{MS}^{2}$ profiles, 108 compounds were putatively identified using the OTCML combined with manual verification, including 12 alkaloids, 49 flavonoids, 13 terpenoids, 14 phenylpropanoids, 4 phenolic acids, 5 phenols, and 11 other phytochemicals. Of these compounds, 17 were confirmed using reference standards. In addition, representative compounds of these different chemical types were used as examples to analyze the fragmentation pathways and characteristic product ions. Moreover, 20 herbs within the QFPD granules were also identified to establish the sources of these chemical components. This is the first rapid profiling of the chemical constituents of QFPD granules using HPLC-Q Exactive Orbitrap-MS and yields valuable information for further quality control and mechanistic studies of QFPD granules.
\end{abstract}

Keywords Chemical constituent identification · Qingfei Paidu granules · HPLC-Q Exactive Orbitrap-MS · Orbitrap traditional Chinese medicine library

\section{Introduction}

Qingfei Paidu (QFPD) granules and decoctions are effective traditional Chinese medicines (TCMs) that are included in the Guidelines for Diagnosis and Treatment of COVID-19 Pneumonia, issued by the National Heath Commission of the

Shuai Fu and Rongrong Cheng authors contributed equally.

Tiangang Liu

liutg@whu.edu.cn

$1 \quad$ Key Laboratory of Combinatorial Biosynthesis and Drug Discovery, Ministry of Education and School of Pharmaceutical Sciences, Wuhan University, Wuhan 430072, China

2 Hubei Engineering Laboratory for Synthetic Microbiology, Wuhan Institute of Biotechnology, Wuhan 430075, China

3 Wuhan Research Center for Infectious Diseases and Cancer, Chinese Academy of Medical Sciences, Wuhan 430072, China
People's Republic of China [1]. QFPD granules and decoctions are based on the following four formulae: MaxingShigan-Tang, Wuling-San, Xiaocaihu-Tang, and SheganMahuang-Tang [2], which are different forms of prescription QFPD. QFPD granules contain 20 herbs: Ephedrae Herba, Glycyrrhizae Radix Et Rhizoma Praeparata Cum Melle, Armeniacae Semen Amarum, Cinnamomi Ramulus, Pogostemonis Herba, Alismatis Rhizoma, Polyporus, Atractylodis Macrocephalae Rhizoma, Poria, Bupleuri Radix, Scutellariae Radix, Pinelliae Rhizoma Praeparatum Cum Zingibere Et Alumine, Zingiberis Rhizoma Recens, Asteris Radix Et Rhizoma, Farfarae Flos, Belamcandae Rhizoma, Asari Radix Et Rhizoma, Dioscoreae Rhizoma, Aurantii Fructus Immaturus, and Citri Reticulatae Pericarpium. In addition, QFPD contains the mineral Gypsum Fibrosum.

In China, QFPD granules and decoctions have been widely used to treat patients infected with SARS-CoV-2 owing to positive treatment results. Early treatment with prescription QFPD was associated with favorable patient outcomes and may be an effective strategy for epidemic control 
[1]. Functional network pharmacology analysis units showed that QFPD protected against COVID-19 through anti-viral and anti-inflammatory activities [2]. A systematic pharmacological study illustrated that QFPD exhibited immune regulation, anti-infection and anti-inflammatory properties, and multi-organ protection [3]. QFPD granules were, therefore, approved for market use by the National Medical Products Administration in China [4]. However, worldwide acceptance of QFPD granules is challenging because of the TCM complexity, and unique theory of treatment, in addition to quality and safety issues $[5,6]$. Thus, comprehensive identification of the chemical components of QFPD granules is extremely critical for quality control, in addition to identification of the active ingredients and investigation of the mechanism-of-action.

Few analytical strategies have been applied to study the chemical constituents of QFPD decoctions, and no detailed analysis of the chemical composition of QFPD granules has been reported [7-9]. Hybrid quadrupole-Orbitrap mass spectrometry (MS) is a powerful tool for structure elucidation of TCMs due to its high resolution and high-quality $\mathrm{MS}^{2}$ fragmentation patterns. In this study, high-performance liquid chromatography (HPLC)-Q Exactive Orbitrap-MS was used to analyze the chemical constituents of QFPD granules, with 108 compounds putatively identified, including 12 alkaloids, 49 flavonoids, 13 terpenoids, 14 phenylpropanoids, 4 phenolic acids, 5 phenols, and 11 other phytochemicals. The individual herbs within the QFPD granules were also analyzed. The aim of this study is to develop an analytical method for elucidating the chemical constituents of QFPD granules and provide valuable quality control and mechanism-of-action data.

\section{Material and Methods}

\section{Reagents and Materials}

QFPD granules were a gift from Renmin Hospital of Wuhan University. The 21 raw materials were purchased from Yifeng Pharmacy Chain Co., Ltd. (Changde, China). Acetonitrile (HPLC grade) and methanol (HPLC grade) were purchased from Merck (Darmstadt, Germany). Formic acid was purchased from Thermo Fisher Scientific (Waltham, MA, USA). Watsons distilled water was obtained from Jingdong Mall (Beijing, China).

Authentic standards of cytosine, sucrose, citric acid, uridine, adenosine, 2-pyrrolidinecarboxylic acid, and guanosine were purchased from Sigma-Aldrich (St. Louis, MO, USA). Nicotinic acid was obtained from Sinopharm Chemical Reagent Co., Ltd. (Shanghai, China). Nicotinamide and tangeretin were purchased from Shanghai Aladdin Bio-Chem Technology Co., Ltd. (Shanghai, China). Salicylic acid was acquired from Ascender Chemical Co., Ltd. (Shanghai, China). Glycyrrhizic acid, 18- $\beta$-glycyrrhetinic acid, isoliquiritigenin, baicalin, and narirutin were purchased from Shanghai Macklin Biochemical Co., Ltd. (Shanghai, China). Chlorogenic acid was a gift from Thermo Fisher Scientific.

\section{Standard Solutions and Sample Preparations}

The QFPD granules were ground, and the resultant powder $(0.4 \mathrm{~g})$ was accurately weighed, dissolved in $60 \%$ methanol $(\mathrm{v} / \mathrm{v} ; 20 \mathrm{~mL})$, and sonicated for $30 \mathrm{~min}$, resulting in partial precipitation of the QFPD granules. The solution was centrifuged, and the supernatant was filtered through a $0.22 \mu \mathrm{m}$ membrane prior to HPLC-Q Exactive Orbitrap-MS.

The individual raw materials were treated using the same procedure.

The authentic standards were dissolved in 50\% methanol and stored at $-80{ }^{\circ} \mathrm{C}$. Prior to qualitative analysis, they were mixed appropriate concentrations and filtered using a $0.22 \mu \mathrm{m}$ membrane.

\section{HPLC-Q Exactive Hybrid Quadrupole-Orbitrap MS}

LC-MS was performed using an UltiMate 3000 UPLC system (Thermo Fisher Scientific), autosampler, a vacuum degasser, binary pump, and column compartment. A Hypersil Gold aQ C18 column $(2.1 \times 150 \mathrm{~mm}, 3 \mu \mathrm{m})$ was used at $40{ }^{\circ} \mathrm{C}$ for chromatography. The mobile phase consisted of acetonitrile/ $0.1 \%$ formic acid (A) and water $/ 0.1 \%$ formic acid (B) at a flow rate of $0.2 \mathrm{~mL} / \mathrm{min}$. The following gradient elution program was used: $0-2 \mathrm{~min}$, 0-5\% (A); 2-42 min, 5-95\% (A); 42-46.9 min, 95\% (A); 46.9-47 min, 95-5\% (A); 47-50 min, 5\% (A). The total run time was $50 \mathrm{~min}$, and the sample injection volume was $5 \mu \mathrm{L}$.

A Q Exactive hybrid quadrupole-Orbitrap mass spectrometer (Thermo Fisher Scientific) with heated electrospray ionization (ESI) was used. Source parameters were optimized with a spray voltage of $3.5 \mathrm{kV}(+) / 3.2 \mathrm{kV}$ $(-)$. The other parameters were set as follows: capillary temperature, $320{ }^{\circ} \mathrm{C}$; auxiliary gas temperature, $350{ }^{\circ} \mathrm{C}$; sheath gas, 40 Arb; auxiliary gas, 15 Arb; sweep gas, 0 Arb; S-lens RF level, 50.

The Orbitrap mass detector was operated in full scan plus data-dependent $\mathrm{MS}^{2}$ mode. The MS resolution was set at 70,000 for the full scan and 17,500 for the $\mathrm{MS}^{2}$ scan. The automatic gain control target and maximum injection time were $1 \times 10^{6}$ ions capacity and $100 \mathrm{~ms}$, respectively. The top $\mathrm{N}$ (N: the number of most abundant ions for fragmentation) was five, while the scan range was $\mathrm{m} / \mathrm{z}$ 100-1500. The normalized collision energies were $20 \%$, 
$40 \%$, and $60 \%$, and the isolation window was $1.2 \mathrm{Da}$. The apex trigger was 5-15 s, and the loop count was 3 . The dynamic exclusion was $5 \mathrm{~s}$.

\section{Data Analysis Using the Orbitrap Traditional Chinese Medicine Library (OTCML) and Manual Verification}

The raw data were imported into the Compound Discoverer (CD) software, which is integrated into the OTCML. The molecular masses, retention times, fragments, and peak areas from both the positive and negative ESI modes were compared to the mzVault library, which was integrated into $\mathrm{CD}$. The mzVault spectral library (Thermo Fisher Scientific) contained the retention times, precise mass ions, and $\mathrm{MS}^{2}$ fragments of 1200 commercial reference standards, which were analyzed using Q Exactive Orbitrap-MS. The software identified peaks with high mass accuracy $(<10 \mathrm{ppm})$ and an isotope pattern variation within $85 \%$. The molecular compositions adhered to the $\mathrm{H} / \mathrm{C}$ ratio rules and were matched to potential compounds using ring and double-bond equivalents. The $\mathrm{MS}^{2}$ profiles were compared with the reference spectra from the mzVault library. Compounds were identified only when the match score was $>85$. In addition, compound identification accuracy was improved by comparing the obtained data and possible fragmentation patterns with those in the literature, and the corresponding individual herb pieces components were analyzed to determine the source of each compound and elucidate chemical compositions.

\section{Results and discussion}

Positive and negative ion modes were used to detect the chemical compounds within the QFPD granules. The base peak chromatograms (BPCs) of the QFPD granules are shown in Fig. 1. In total, 108 compounds are putatively identified (Table 1). The BPCs of the individual herb pieces are shown in Figs. S1 and S2. Compound identification is summarized below.

\section{Alkaloids}

Twelve alkaloids were detected. Compounds 19, 20, 21, 15, and $\mathbf{1 6}$ are observed in the positive BPC of QFPD, with no matching identification results after data processing using the OTCML. The mass spectra of compounds 15, 19, and 21 display the same fragment ions at $m / z, 117.0701$ (Fig. S3). The mass spectra of compounds 19 and 20 exhibit the same $[\mathrm{M}+\mathrm{H}]^{+}$ions at $m / z 166.1226\left(\mathrm{C}_{10} \mathrm{H}_{15} \mathrm{NO}\right)$, with the same fragment ions also observed at $m / z 148.1120[\mathrm{M}+\mathrm{H}$
Fig. 1 Base peak chromatograms of QFPD granules obtained using high performance liquid chromatographyQ Exactive hybrid quadrupoleOrbitrap mass spectrometry. A Electrospray ionization in the positive mode $(\operatorname{ESI}(+)), \mathbf{B}$ electrospray ionization in the negative mode $(\operatorname{ESI}(-))$
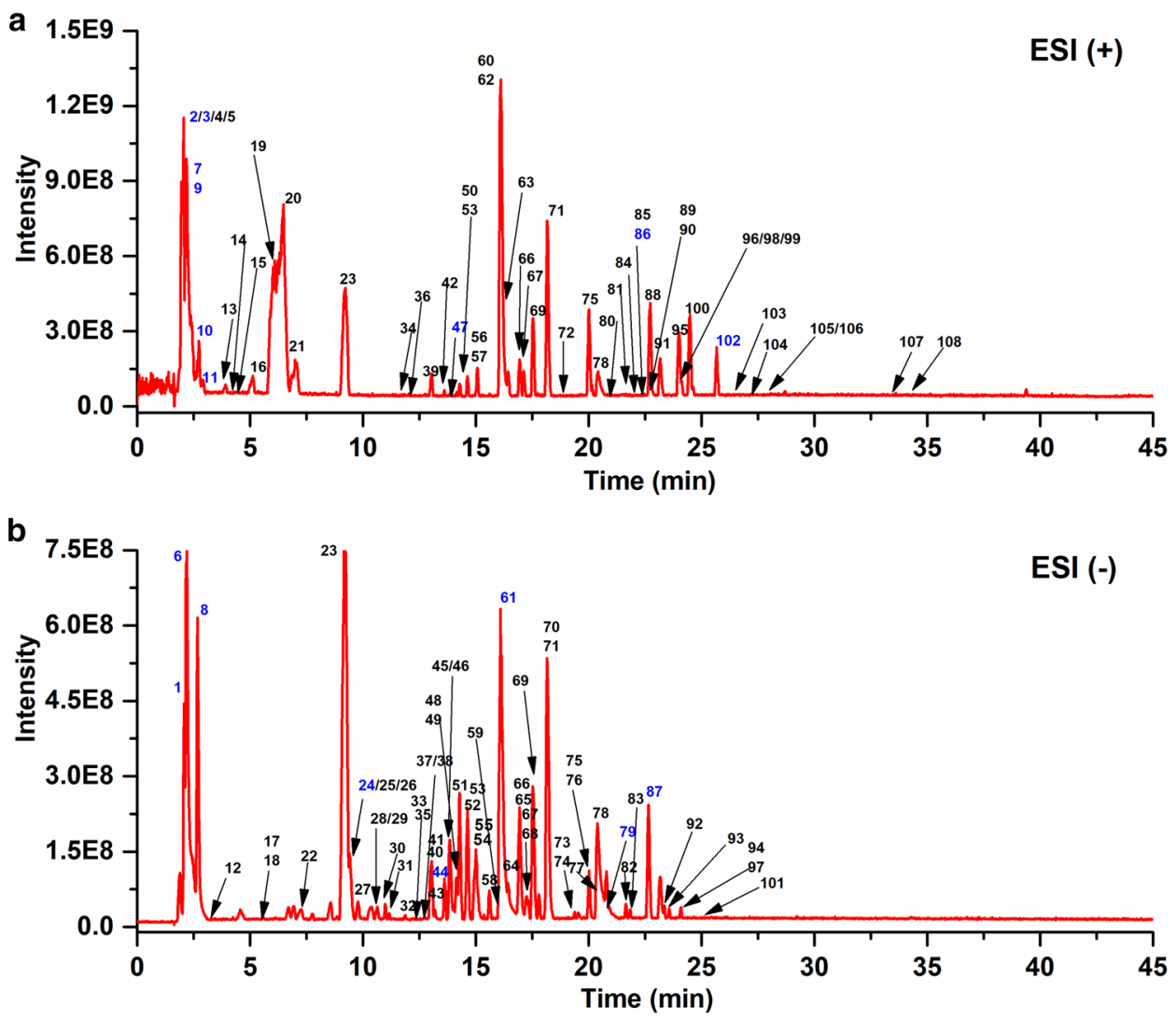
Table 1 Identification of the chemical components of QFPD granules using high performance liquid chromatography-Q Exactive hybrid quadrupole-Orbitrap mass spectrometry combined with the Orbitrap traditional Chinese medicine library

\begin{tabular}{|c|c|c|c|c|c|c|c|c|}
\hline No. & $\mathrm{RT}(\min )$ & Formula & Potential compound & Detected $\mathrm{m} / \mathrm{z}$ & Characterized $\mathrm{MS}^{2}$ & Compound class & Herb & Refs \\
\hline $1^{\mathrm{d}}$ & 2.11 & $\mathrm{C}_{12} \mathrm{H}_{22} \mathrm{O}_{11}$ & Sucrose & $341.1069[\mathrm{M}-\mathrm{H}]^{-}$ & $\begin{array}{l}89.0235 \\
71.1031 \\
59.0132^{b}\end{array}$ & Miscellaneous & $P R$ & [10] \\
\hline $2^{\mathrm{d}}$ & 2.12 & $\mathrm{C}_{5} \mathrm{H}_{9} \mathrm{NO}_{2}$ & 2-Pyrrolidinecarboxylic acid & $116.0709[\mathrm{M}+\mathrm{H}]^{+}$ & $\begin{array}{l}116.0708 \\
70.0658^{b}\end{array}$ & Miscellaneous & $D R$ & [11] \\
\hline $3^{\mathrm{d}}$ & 2.12 & $\mathrm{C}_{4} \mathrm{H}_{5} \mathrm{~N}_{3} \mathrm{O}$ & Cytosine & $112.0508[\mathrm{M}+\mathrm{H}]^{+}$ & $\begin{array}{l}112.0508^{\mathrm{b}} \\
95.0244\end{array}$ & Alkaloids & $A R$ & \\
\hline 4 & 2.13 & $\mathrm{C}_{5} \mathrm{H}_{11} \mathrm{NO}_{2}$ & Betaine & $118.0865[\mathrm{M}+\mathrm{H}]^{+}$ & $\begin{array}{l}118.0864^{\mathrm{b}} \\
59.0737\end{array}$ & Alkaloids & $A E$ & \\
\hline 5 & 2.14 & $\mathrm{C}_{7} \mathrm{H}_{7} \mathrm{NO}_{2}$ & Trigonelline & $138.0550[\mathrm{M}+\mathrm{H}]^{+}$ & $\begin{array}{l}138.0550^{b} \\
110.0603 \\
94.0656\end{array}$ & Alkaloids & $P C$ & [12] \\
\hline $6^{\mathrm{d}}$ & 2.2 & $\mathrm{C}_{6} \mathrm{H}_{8} \mathrm{O}_{7}$ & Citric acid & $191.0185[\mathrm{M}-\mathrm{H}]^{-}$ & $\begin{array}{l}111.0078^{b} \\
87.0079\end{array}$ & Miscellaneous & $G R$ & \\
\hline $7^{\mathrm{d}}$ & 2.66 & $\mathrm{C}_{6} \mathrm{H}_{5} \mathrm{NO}_{2}$ & Nicotinic acid & $124.0395[\mathrm{M}+\mathrm{H}]^{+}$ & $\begin{array}{l}124.0394^{b} \\
96.0448 \\
80.05\end{array}$ & Alkaloids & & \\
\hline $8^{\text {acd }}$ & 2.69 & $\mathrm{C}_{9} \mathrm{H}_{12} \mathrm{~N}_{2} \mathrm{O}_{6}$ & Uridine & $243.0608[\mathrm{M}-\mathrm{H}]^{-}$ & $\begin{array}{l}200.0554 \\
152.0339 \\
122.0234 \\
110.0238^{b}\end{array}$ & Miscellaneous & $P R$ & [13] \\
\hline $9^{d}$ & 2.69 & $\mathrm{C}_{6} \mathrm{H}_{6} \mathrm{~N}_{2} \mathrm{O}$ & Nicotinamide & $123.0555[\mathrm{M}+\mathrm{H}]^{+}$ & $\begin{array}{l}123.0554^{b} \\
96.0448 \\
80.0501\end{array}$ & Alkaloids & $F F$ & \\
\hline $10^{\mathrm{ad}}$ & 2.73 & $\mathrm{C}_{10} \mathrm{H}_{13} \mathrm{~N}_{5} \mathrm{O}_{4}$ & Adenosine & $268.1041[\mathrm{M}+\mathrm{H}]^{+}$ & $136.0618^{\mathrm{b}}$ & Miscellaneous & $P C$ & [14] \\
\hline $11^{\text {acd }}$ & 2.87 & $\mathrm{C}_{10} \mathrm{H}_{13} \mathrm{~N}_{5} \mathrm{O}_{5}$ & Guanosine & $284.0989[\mathrm{M}+\mathrm{H}]^{+}$ & $152.0567^{\mathrm{b}}$ & Miscellaneous & $P C$ & [14] \\
\hline 12 & 3.41 & $\mathrm{C}_{7} \mathrm{H}_{6} \mathrm{O}_{5}$ & Gallic acid & $169.013[\mathrm{M}-\mathrm{H}]^{-}$ & $\begin{array}{l}125.0233^{b} \\
97.0285 \\
69.0337\end{array}$ & Phenolic acids & & \\
\hline 13 & 3.9 & $\mathrm{C}_{9} \mathrm{H}_{11} \mathrm{NO}_{2}$ & L-Phenylalanine & $166.0863[\mathrm{M}+\mathrm{H}]^{+}$ & $\begin{array}{l}120.0809^{b} \\
103.0546\end{array}$ & Miscellaneous & & \\
\hline 14 & 4.22 & $\mathrm{C}_{6} \mathrm{H}_{6} \mathrm{O}_{3}$ & 5-Hydroxymethylfurfural & $127.0392[\mathrm{M}+\mathrm{H}]^{+}$ & $\begin{array}{l}109.0288^{b} \\
81.0341\end{array}$ & Miscellaneous & $P C$ & [15] \\
\hline 15 & 4.68 & $\mathrm{C}_{9} \mathrm{H}_{13} \mathrm{NO}$ & L-norephedrine & $152.1069[\mathrm{M}+\mathrm{H}]^{+}$ & $\begin{array}{l}134.0965^{\mathrm{b}} \\
117.0701\end{array}$ & Alkaloids & $E H$ & [16] \\
\hline 16 & 5.12 & $\mathrm{C}_{9} \mathrm{H}_{13} \mathrm{NO}$ & D-norpseudoephedrine & $152.1069[\mathrm{M}+\mathrm{H}]^{+}$ & $\begin{array}{l}134.0965^{\mathrm{b}} \\
117.0701\end{array}$ & Alkaloids & $E H$ & [16] \\
\hline 17 & 5.24 & $\mathrm{C}_{15} \mathrm{H}_{14} \mathrm{O}_{7}$ & $(-)$-Gallocatechin & $305.0651[\mathrm{M}-\mathrm{H}]^{-}$ & $\begin{array}{l}219.0654 \\
137.0232 \\
125.0232^{b}\end{array}$ & Phenols & & \\
\hline 18 & 5.32 & $\mathrm{C}_{7} \mathrm{H}_{6} \mathrm{O}_{4}$ & Protocatechuic acid & $153.0181[\mathrm{M}-\mathrm{H}]^{-}$ & $109.0284^{\mathrm{b}}$ & Phenolic acids & $G R$ & [15] \\
\hline 19 & 6.09 & $\mathrm{C}_{10} \mathrm{H}_{15} \mathrm{NO}$ & L-ephedrine & $166.1226[\mathrm{M}+\mathrm{H}]^{+}$ & $\begin{array}{l}148.1120^{\mathrm{b}} \\
133.0887 \\
117.0701 \\
91.0547\end{array}$ & Alkaloids & $E H$ & [16] \\
\hline 20 & 6.47 & $\mathrm{C}_{10} \mathrm{H}_{15} \mathrm{NO}$ & D-pseudoephedrine & $166.1226[\mathrm{M}+\mathrm{H}]^{+}$ & $\begin{array}{l}148.1120^{\mathrm{b}} \\
133.0887 \\
117.0701 \\
91.0547\end{array}$ & Alkaloids & $E H$ & [16] \\
\hline 21 & 6.99 & $\mathrm{C}_{11} \mathrm{H}_{17} \mathrm{NO}$ & Methylephedrine & $180.1382[\mathrm{M}+\mathrm{H}]^{+}$ & $\begin{array}{l}162.1276^{\mathrm{b}} \\
147.1041 \\
135.0805 \\
117.0701\end{array}$ & Alkaloids & $E H$ & [16] \\
\hline 22 & 7.26 & $\mathrm{C}_{7} \mathrm{H}_{6} \mathrm{O}_{3}$ & Protocatechualdehyde & $137.0233[\mathrm{M}-\mathrm{H}]^{-}$ & $\begin{array}{l}137.0233^{b} \\
119.0126 \\
109.0285\end{array}$ & Phenols & $C R$ & [15] \\
\hline
\end{tabular}


Table 1 (continued)

\begin{tabular}{|c|c|c|c|c|c|c|c|c|}
\hline No. & RT (min) & Formula & Potential compound & Detected $\mathrm{m} / \mathrm{z}$ & Characterized $\mathrm{MS}^{2}$ & Compound class & Herb & Refs \\
\hline $23^{c}$ & 9.16 & $\mathrm{C}_{20} \mathrm{H}_{27} \mathrm{NO}_{11}$ & Amygdalin & $456.1492[\mathrm{M}-\mathrm{H}]^{-}$ & $\begin{array}{l}323.0963 \\
221.0653 \\
161.0443 \\
59.0132^{b}\end{array}$ & Miscellaneous & $A S$ & [17] \\
\hline $24^{\mathrm{cd}}$ & 9.43 & $\mathrm{C}_{16} \mathrm{H}_{18} \mathrm{O}_{9}$ & Chlorogenic acid & $353.0862[\mathrm{M}-\mathrm{H}]^{-}$ & $\begin{array}{l}191.0548^{b} \\
135.0441 \\
179.0337\end{array}$ & Phenylpropanoids & $F F$ & [18] \\
\hline 25 & 9.46 & $\mathrm{C}_{9} \mathrm{H}_{6} \mathrm{O}_{4}$ & Esculetin & $177.0181[\mathrm{M}-\mathrm{H}]^{-}$ & $\begin{array}{l}177.0180^{\mathrm{b}} \\
149.0236 \\
133.0284 \\
105.0336\end{array}$ & Phenylpropanoids & & \\
\hline 26 & 9.48 & $\mathrm{C}_{7} \mathrm{H}_{6} \mathrm{O}_{2}$ & $p$-Hydroxybenzaldehyde & $121.0285[\mathrm{M}-\mathrm{H}]^{-}$ & $\begin{array}{l}121.0284^{b} \\
93.0336\end{array}$ & Phenols & & \\
\hline 27 & 9.78 & $\mathrm{C}_{9} \mathrm{H}_{8} \mathrm{O}_{4}$ & Caffeic acid & $179.0337[\mathrm{M}-\mathrm{H}]^{-}$ & $135.0441^{\mathrm{b}}$ & Phenolic acids & & \\
\hline $28^{\mathrm{a}}$ & 10.49 & $\mathrm{C}_{15} \mathrm{H}_{14} \mathrm{O}_{6}$ & Catechin hydrate & $289.0703[\mathrm{M}-\mathrm{H}]^{-}$ & $\begin{array}{l}245.0805 \\
123.044 \\
109.0284^{b}\end{array}$ & Flavonoids & & \\
\hline $29^{\mathrm{a}}$ & 10.63 & $\mathrm{C}_{15} \mathrm{H}_{12} \mathrm{O}_{7}$ & Taxifolin & $303.0494[\mathrm{M}-\mathrm{H}]^{-}$ & $\begin{array}{l}177.018 \\
125.0233^{\mathrm{b}}\end{array}$ & Flavonoids & $S R$ & [19] \\
\hline $30^{c}$ & 10.98 & $\mathrm{C}_{27} \mathrm{H}_{30} \mathrm{O}_{15}$ & Vicenin II & $593.1482[\mathrm{M}-\mathrm{H}]^{-}$ & $\begin{array}{l}353.0648^{\mathrm{b}} \\
383.0753 \\
473.1062 \\
297.075\end{array}$ & Flavonoids & $G R$ & {$[8]$} \\
\hline 31 & 11.12 & $\mathrm{C}_{25} \mathrm{H}_{24} \mathrm{O}_{12}$ & 1,3-Dicaffeoylquinic acid & $515.1168[\mathrm{M}-\mathrm{H}]^{-}$ & $\begin{array}{l}353.0859 \\
191.0547^{\mathrm{b}} \\
179.0336 \\
135.044\end{array}$ & Phenylpropanoids & & \\
\hline 32 & 11.87 & $\mathrm{C}_{9} \mathrm{H}_{8} \mathrm{O}_{3}$ & $p$-Coumaric acid & $163.0400[\mathrm{M}-\mathrm{H}]^{-}$ & $\begin{array}{l}119.0496^{\mathrm{b}} \\
163.0394\end{array}$ & Phenylpropanoids & & \\
\hline $33^{\mathrm{ac}}$ & 11.95 & $\mathrm{C}_{26} \mathrm{H}_{28} \mathrm{O}_{14}$ & Isoschaftoside & $563.1376[\mathrm{M}-\mathrm{H}]^{-}$ & $\begin{array}{l}353.0648^{b} \\
383.0754 \\
473.1073\end{array}$ & Flavonoids & $G R$ & {$[20]$} \\
\hline 34 & 11.96 & $\mathrm{C}_{9} \mathrm{H}_{10} \mathrm{O}_{4}$ & $\begin{array}{l}\text { 3,5-Dimethoxy-4-hydroxyben- } \\
\text { zaldehyde }\end{array}$ & $183.0652[\mathrm{M}+\mathrm{H}]^{+}$ & $\begin{array}{l}140.0469 \\
123.0443 \\
95.0497^{\mathrm{b}}\end{array}$ & Phenols & $\begin{array}{l}C R \\
E H \\
R E\end{array}$ & [15] \\
\hline 35 & 12.18 & $\mathrm{C}_{21} \mathrm{H}_{20} \mathrm{O}_{11}$ & Orientin & $447.0913[\mathrm{M}-\mathrm{H}]^{-}$ & $\begin{array}{l}357.06 \\
327.0496^{\mathrm{b}} \\
299.0541 \\
133.028\end{array}$ & Alkaloids & $C P$ & [21] \\
\hline 36 & 12.6 & $\mathrm{C}_{10} \mathrm{H}_{8} \mathrm{O}_{4}$ & Scopoletin & $193.0497[\mathrm{M}+\mathrm{H}]^{+}$ & $\begin{array}{l}193.0496^{\mathrm{b}} \\
178.026 \\
133.0285\end{array}$ & Phenylpropanoids & $A F$ & [22] \\
\hline $37^{\mathrm{ac}}$ & 12.7 & $\mathrm{C}_{26} \mathrm{H}_{30} \mathrm{O}_{13}$ & $\begin{array}{l}\text { Naringenin 7-O-(2- } \beta- \\
\text { D-apiofuranosyl)- } \beta \text {-D- } \\
\text { glucopyranoside }\end{array}$ & $549.1588[\mathrm{M}-\mathrm{H}]^{-}$ & $\begin{array}{l}255.0649 \\
135.0077 \\
119.0492^{\mathrm{b}}\end{array}$ & Flavonoids & $G R$ & [20] \\
\hline 38 & 12.84 & $\mathrm{C}_{10} \mathrm{H}_{10} \mathrm{O}_{4}$ & Ferulic acid & $193.0492[\mathrm{M}-\mathrm{H}]^{-}$ & $\begin{array}{l}178.0258 \\
134.0362^{b}\end{array}$ & Phenylpropanoids & & \\
\hline 39 & 13.03 & $\mathrm{C}_{11} \mathrm{H}_{10} \mathrm{O}_{5}$ & Isofraxidin & $223.0601[\mathrm{M}+\mathrm{H}]^{+}$ & $\begin{array}{l}223.0601^{\mathrm{b}} \\
190.0261 \\
162.0311\end{array}$ & Phenylpropanoids & & \\
\hline $40^{c}$ & 13.04 & $\mathrm{C}_{27} \mathrm{H}_{32} \mathrm{O}_{15}$ & Eriocitrin & $595.1638[\mathrm{M}-\mathrm{H}]^{-}$ & $\begin{array}{l}459.1152 \\
151.0025^{\mathrm{b}} \\
135.0441\end{array}$ & Flavonoids & $\begin{array}{l}C P \\
A F\end{array}$ & $\begin{array}{l}{[21]} \\
{[23]}\end{array}$ \\
\hline $41^{\mathrm{c}}$ & 13.04 & $\mathrm{C}_{26} \mathrm{H}_{30} \mathrm{O}_{13}$ & Liquiritin apioside & $549.1586[\mathrm{M}-\mathrm{H}]^{-}$ & $\begin{array}{l}119.0491^{\mathrm{b}} \\
135.0077 \\
255.0649\end{array}$ & Flavonoids & $G R$ & [20] \\
\hline
\end{tabular}


Table 1 (continued)

\begin{tabular}{|c|c|c|c|c|c|c|c|c|}
\hline No. & RT (min) & Formula & Potential compound & Detected $\mathrm{m} / \mathrm{z}$ & Characterized $\mathrm{MS}^{2}$ & Compound class & Herb & Refs \\
\hline $42^{c}$ & 13.18 & $\mathrm{C}_{27} \mathrm{H}_{30} \mathrm{O}_{16}$ & Rutin & $609.1431[\mathrm{M}-\mathrm{H}]^{-}$ & $\begin{array}{l}300.0258^{b} \\
271.0234 \\
255.0284\end{array}$ & Flavonoids & & \\
\hline 43 & 13.39 & $\mathrm{C}_{9} \mathrm{H}_{6} \mathrm{O}_{4}$ & 5,7-Dihydroxychromone & $177.0180[\mathrm{M}-\mathrm{H}]^{-}$ & $\begin{array}{l}177.0180^{\mathrm{b}} \\
135.0076\end{array}$ & Flavonoids & & \\
\hline $44^{\mathrm{d}}$ & 13.75 & $\mathrm{C}_{7} \mathrm{H}_{6} \mathrm{O}_{3}$ & Salicylic acid & $137.0233[\mathrm{M}-\mathrm{H}]^{-}$ & $\begin{array}{l}137.0233 \\
93.0337^{\mathrm{b}}\end{array}$ & Phenolic acids & $A E$ & {$[15]$} \\
\hline 45 & 13.8 & $\mathrm{C}_{14} \mathrm{H}_{12} \mathrm{O}_{4}$ & Piceatannol & $243.0648[\mathrm{M}-\mathrm{H}]^{-}$ & $\begin{array}{l}243.0648^{b} \\
201.0544 \\
159.0439\end{array}$ & Phenols & & \\
\hline 46 & 13.84 & $\mathrm{C}_{25} \mathrm{H}_{24} \mathrm{O}_{12}$ & Isochlorogenic acid B & $515.1165[\mathrm{M}-\mathrm{H}]^{-}$ & $\begin{array}{l}353.0856 \\
191.0547 \\
179.0336 \\
135.0440^{b}\end{array}$ & Phenylpropanoids & $F F$ & {$[18]$} \\
\hline $47^{\mathrm{cd}}$ & 13.89 & $\mathrm{C}_{27} \mathrm{H}_{32} \mathrm{O}_{14}$ & Narirutin & $581.1863[\mathrm{M}+\mathrm{H}]^{+}$ & $\begin{array}{l}273.0755^{\mathrm{b}} \\
153.0181 \\
85.0289 \\
71.0498\end{array}$ & Flavonoids & $C P$ & {$[21]$} \\
\hline $48^{\mathrm{a}}$ & 14.02 & $\mathrm{C}_{29} \mathrm{H}_{36} \mathrm{O}_{15}$ & Verbascoside & $623.1945[\mathrm{M}-\mathrm{H}]^{-}$ & $\begin{array}{l}461.1639 \\
161.0231^{\mathrm{b}} \\
133.0283\end{array}$ & Phenylpropanoids & $P H$ & {$[15]$} \\
\hline 49 & 14.13 & $\mathrm{C}_{25} \mathrm{H}_{24} \mathrm{O}_{12}$ & 3,5-Dicaffeoylquinic acid & $515.1165[\mathrm{M}-\mathrm{H}]^{-}$ & $\begin{array}{l}353.0878 \\
191.0558^{\mathrm{b}} \\
179.0346 \\
135.0448\end{array}$ & Phenylpropanoids & $F F$ & {$[18]$} \\
\hline 50 & 14.14 & $\mathrm{C}_{22} \mathrm{H}_{22} \mathrm{O}_{11}$ & Tectoridin & $463.1234[\mathrm{M}+\mathrm{H}]^{+}$ & $\begin{array}{l}301.0705^{\mathrm{b}} \\
286.047\end{array}$ & Flavonoids & $B H$ & $\begin{array}{l}{[24]} \\
{[25]}\end{array}$ \\
\hline $51^{\mathrm{ac}}$ & 14.27 & $\mathrm{C}_{27} \mathrm{H}_{32} \mathrm{O}_{14}$ & Naringin & $579.1688[\mathrm{M}-\mathrm{H}]^{-}$ & $\begin{array}{l}271.0597 \\
151.0025^{b} \\
119.0491 \\
107.0129\end{array}$ & Flavonoids & $\begin{array}{l}A F \\
C P\end{array}$ & $\begin{array}{l}{[23]} \\
{[21]}\end{array}$ \\
\hline 52 & 14.57 & $\mathrm{C}_{9} \mathrm{H}_{16} \mathrm{O}_{4}$ & Azelaic acid & $187.0962[\mathrm{M}-\mathrm{H}]^{-}$ & $\begin{array}{l}125.0960^{\mathrm{b}} \\
97.0649\end{array}$ & Miscellaneous & & \\
\hline 53 & 14.62 & $\mathrm{C}_{28} \mathrm{H}_{34} \mathrm{O}_{15}$ & Neohesperidin & $609.1796[\mathrm{M}-\mathrm{H}]^{-}$ & $\begin{array}{l}609.1791 \\
301.0700^{b} \\
286.0466\end{array}$ & Flavonoids & $C P$ & \\
\hline 54 & 14.95 & $\mathrm{C}_{25} \mathrm{H}_{24} \mathrm{O}_{12}$ & Isochlorogenic acid C & $515.1166[\mathrm{M}-\mathrm{H}]^{-}$ & $\begin{array}{l}353.088 \\
191.0558 \\
173.0452 \\
135.0448^{b}\end{array}$ & Phenylpropanoids & $F F$ & {$[18]$} \\
\hline $55^{\mathrm{c}}$ & 15 & $\mathrm{C}_{28} \mathrm{H}_{34} \mathrm{O}_{15}$ & Hesperidin & $609.1796[\mathrm{M}-\mathrm{H}]^{-}$ & $\begin{array}{l}609.1791 \\
301.0699^{b} \\
286.0466\end{array}$ & Flavonoids & $C P$ & {$[26]$} \\
\hline 56 & 15.06 & $\mathrm{C}_{24} \mathrm{H}_{26} \mathrm{O}_{13}$ & Iridin & $523.1445[\mathrm{M}+\mathrm{H}]^{+}$ & $\begin{array}{l}361.0915^{\mathrm{b}} \\
346.0679 \\
331.0445\end{array}$ & Flavonoids & $B H$ & {$[25]$} \\
\hline 57 & 15.06 & $\mathrm{C}_{9} \mathrm{H}_{6} \mathrm{O}_{2}$ & Coumarin & $147.0440[\mathrm{M}+\mathrm{H}]^{+}$ & $\begin{array}{l}147.0440^{b} \\
103.0546 \\
91.0547\end{array}$ & Phenylpropanoids & $\begin{array}{l}C R \\
E H\end{array}$ & [15] \\
\hline $58^{\mathrm{a}}$ & 15.63 & $\mathrm{C}_{15} \mathrm{H}_{12} \mathrm{O}_{6}$ & Eriodictyol & $287.0547[\mathrm{M}-\mathrm{H}]^{-}$ & $\begin{array}{l}287.0547 \\
161.0231 \\
125.0233^{b}\end{array}$ & Flavonoids & $A F$ & [23] \\
\hline $59^{c}$ & 15.86 & $\mathrm{C}_{26} \mathrm{H}_{30} \mathrm{O}_{13}$ & Isoliquiritin apioside & $549.1589[\mathrm{M}-\mathrm{H}]^{-}$ & $\begin{array}{l}255.0649 \\
153.0181 \\
135.0077 \\
119.0491^{\mathrm{b}}\end{array}$ & Flavonoids & $G R$ & {$[20]$} \\
\hline
\end{tabular}


Table 1 (continued)

\begin{tabular}{|c|c|c|c|c|c|c|c|c|}
\hline No. & $\mathrm{RT}(\min )$ & Formula & Potential compound & Detected $\mathrm{m} / \mathrm{z}$ & Characterized $\mathrm{MS}^{2}$ & Compound class & Herb & Refs \\
\hline 60 & 16.06 & $\mathrm{C}_{22} \mathrm{H}_{22} \mathrm{O}_{9}$ & Ononin & $431.1336[\mathrm{M}+\mathrm{H}]^{+}$ & $\begin{array}{l}269.0807^{b} \\
254.0573 \\
237.0544\end{array}$ & Flavonoids & $G R$ & [27] \\
\hline $61^{\mathrm{cd}}$ & 16.08 & $\mathrm{C}_{21} \mathrm{H}_{18} \mathrm{O}_{11}$ & Baicalin & $445.0753[\mathrm{M}-\mathrm{H}]^{-}$ & $269.0439^{\mathrm{b}}$ & Flavonoids & $S R$ & [19] \\
\hline 62 & 16.15 & $\mathrm{C}_{21} \mathrm{H}_{20} \mathrm{O}_{10}$ & Oroxin A & $433.1129[\mathrm{M}+\mathrm{H}]^{+}$ & $\begin{array}{l}271.0599^{b} \\
253.0493 \\
123.0078\end{array}$ & Flavonoids & $S R$ & [19] \\
\hline $63^{\mathrm{a}}$ & 16.23 & $\mathrm{C}_{21} \mathrm{H}_{22} \mathrm{O}_{9}$ & Isoliquiritin & $419.1334[\mathrm{M}+\mathrm{H}]^{+}$ & $\begin{array}{l}257.0806^{\mathrm{b}} \\
147.0439 \\
137.0232\end{array}$ & Flavonoids & $G R$ & [20] \\
\hline $64^{\mathrm{c}}$ & 16.51 & $\mathrm{C}_{15} \mathrm{H}_{12} \mathrm{O}_{4}$ & Liquiritigenin & $255.0651[\mathrm{M}-\mathrm{H}]^{-}$ & $\begin{array}{l}135.0078 \\
119.0492^{\mathrm{b}} \\
91.0181\end{array}$ & Flavonoids & $G R$ & [20] \\
\hline 65 & 16.87 & $\mathrm{C}_{11} \mathrm{H}_{6} \mathrm{O}_{4}$ & Bergaptol & $201.0180[\mathrm{M}-\mathrm{H}]^{-}$ & $\begin{array}{l}201.0192^{b} \\
183.1012 \\
139.1117\end{array}$ & Phenylpropanoids & & \\
\hline $66^{c}$ & 16.92 & $\mathrm{C}_{21} \mathrm{H}_{18} \mathrm{O}_{11}$ & Norwogonin-8-glucuronide & $445.0753[\mathrm{M}-\mathrm{H}]^{-}$ & $269.0441^{\mathrm{b}}$ & Flavonoids & $S R$ & [19] \\
\hline 67 & 17.2 & $\mathrm{C}_{28} \mathrm{H}_{34} \mathrm{O}_{14}$ & Poncirin & $593.1842[\mathrm{M}-\mathrm{H}]^{-}$ & $\begin{array}{l}593.184 \\
285.0753^{\mathrm{b}} \\
151.0024\end{array}$ & Flavonoids & $A F$ & [23] \\
\hline 68 & 17.24 & $\mathrm{C}_{21} \mathrm{H}_{18} \mathrm{O}_{11}$ & Norwogonin-7-glucuronide & $445.0752[\mathrm{M}-\mathrm{H}]^{-}$ & $269.0439^{\mathrm{b}}$ & Flavonoids & $S R$ & [19] \\
\hline $69^{c}$ & 17.51 & $\mathrm{C}_{22} \mathrm{H}_{20} \mathrm{O}_{11}$ & $\begin{array}{l}\text { Oroxylin A-7-O- } \beta-\mathrm{D}- \\
\text { glucuronide }\end{array}$ & $459.0910[\mathrm{M}-\mathrm{H}]^{-}$ & $\begin{array}{l}283.0595 \\
268.0362^{\mathrm{b}}\end{array}$ & Flavonoids & $S R$ & [19] \\
\hline $70^{\mathrm{c}}$ & 18.11 & $\mathrm{C}_{21} \mathrm{H}_{18} \mathrm{O}_{11}$ & Baicalein-6-glucuronide & $445.0754[\mathrm{M}-\mathrm{H}]^{-}$ & $269.0441^{\mathrm{b}}$ & Flavonoids & $S R$ & [19] \\
\hline $71^{\mathrm{c}}$ & 18.14 & $\mathrm{C}_{22} \mathrm{H}_{20} \mathrm{O}_{11}$ & Wogonoside & $459.0909[\mathrm{M}-\mathrm{H}]^{-}$ & $\begin{array}{l}283.0595 \\
268.0362^{b}\end{array}$ & Flavonoids & $S R$ & [19] \\
\hline 72 & 18.58 & $\mathrm{C}_{15} \mathrm{H}_{12} \mathrm{O}_{5}$ & Naringenin chalcone & $273.0756[\mathrm{M}+\mathrm{H}]^{+}$ & $\begin{array}{l}273.0757 \\
153.0182^{\mathrm{b}} \\
147.044 \\
119.0493\end{array}$ & Flavonoids & $\begin{array}{l}G R \\
A F \\
C P\end{array}$ & $\begin{array}{l}{[20]} \\
{[23]} \\
{[21]}\end{array}$ \\
\hline $73^{c}$ & 19.32 & $\mathrm{C}_{16} \mathrm{H}_{14} \mathrm{O}_{6}$ & Hesperetin & $301.0702[\mathrm{M}-\mathrm{H}]^{-}$ & $\begin{array}{l}301.0702^{\mathrm{b}} \\
286.044 \\
164.0103 \\
108.0207\end{array}$ & Flavonoids & $C P$ & [26] \\
\hline 74 & 19.38 & $\mathrm{C}_{16} \mathrm{H}_{12} \mathrm{O}_{6}$ & Tectorigenin & $299.0546[\mathrm{M}-\mathrm{H}]^{-}$ & $\begin{array}{l}284.0301^{\mathrm{b}} \\
240.0414\end{array}$ & Flavonoids & & \\
\hline $75^{\mathrm{ac}}$ & 20.01 & $\mathrm{C}_{18} \mathrm{H}_{16} \mathrm{O}_{8}$ & Irigenin & $359.0756[\mathrm{M}-\mathrm{H}]^{-}$ & $\begin{array}{l}344.0519 \\
329.0286^{\mathrm{b}} \\
314.0054 \\
286.0104\end{array}$ & Flavonoids & $B H$ & [24] \\
\hline 76 & 20.03 & $\mathrm{C}_{16} \mathrm{H}_{12} \mathrm{O}_{7}$ & Isorhamnetin & $315.0496[\mathrm{M}-\mathrm{H}]^{-}$ & $\begin{array}{l}315.0496 \\
300.0260^{\mathrm{b}} \\
271.0237 \\
151.002\end{array}$ & Flavonoids & $A E$ & [28] \\
\hline $77^{\mathrm{c}}$ & 20.15 & $\mathrm{C}_{17} \mathrm{H}_{14} \mathrm{O}_{7}$ & Iristectorigenin B & $329.0652[\mathrm{M}-\mathrm{H}]^{-}$ & $\begin{array}{l}314.0417 \\
299.0180^{b} \\
271.0235\end{array}$ & Flavonoids & $B H$ & [24] \\
\hline 78 & 20.39 & $\mathrm{C}_{15} \mathrm{H}_{10} \mathrm{O}_{5}$ & Baicalein & $269.0443[\mathrm{M}-\mathrm{H}]^{-}$ & $\begin{array}{l}269.0457^{b} \\
241.0507 \\
223.0398\end{array}$ & Flavonoids & $S R$ & [19] \\
\hline $79^{\mathrm{cd}}$ & 20.97 & $\mathrm{C}_{15} \mathrm{H}_{12} \mathrm{O}_{4}$ & Isoliquiritigenin & $255.0650[\mathrm{M}-\mathrm{H}]^{-}$ & $\begin{array}{l}135.0076 \\
119.0491^{\mathrm{b}} \\
91.018\end{array}$ & Flavonoids & $G R$ & [20] \\
\hline 80 & 21.08 & $\mathrm{C}_{16} \mathrm{H}_{12} \mathrm{O}_{4}$ & Formononetin & $269.0807[\mathrm{M}+\mathrm{H}]^{+}$ & $\begin{array}{l}269.0807^{\mathrm{b}} \\
254.0574\end{array}$ & Flavonoids & $G R$ & [20] \\
\hline
\end{tabular}


Table 1 (continued)

\begin{tabular}{|c|c|c|c|c|c|c|c|c|}
\hline No. & RT (min) & Formula & Potential compound & Detected $\mathrm{m} / \mathrm{z}$ & Characterized $\mathrm{MS}^{2}$ & Compound class & Herb & Refs \\
\hline $81^{\mathrm{ac}}$ & 21.35 & $\mathrm{C}_{15} \mathrm{H}_{16} \mathrm{O}_{4}$ & Isomeranzin & $261.1119[\mathrm{M}+\mathrm{H}]^{+}$ & $\begin{array}{l}189.0546^{\mathrm{b}} \\
159.0439 \\
131.0492\end{array}$ & Phenylpropanoids & & \\
\hline $82^{\mathrm{ac}}$ & 21.65 & $\mathrm{C}_{42} \mathrm{H}_{62} \mathrm{O}_{17}$ & Licorice-saponin G2 & $837.3869[\mathrm{M}-\mathrm{H}]^{-}$ & $\begin{array}{l}837.386 \\
351.0552 \\
193.0341 \\
113.0235^{\mathrm{b}}\end{array}$ & Terpenoids & $G R$ & [20] \\
\hline $83^{c}$ & 21.83 & $\mathrm{C}_{26} \mathrm{H}_{30} \mathrm{O}_{8}$ & Limonin & $469.1846[\mathrm{M}-\mathrm{H}]^{-}$ & $\begin{array}{l}469.1831^{b} \\
249.0909 \\
229.1214\end{array}$ & Terpenoids & $A F$ & [22] \\
\hline 84 & 21.83 & $\mathrm{C}_{15} \mathrm{H}_{22} \mathrm{O}_{2}$ & Curcumenol & $235.1693[\mathrm{M}+\mathrm{H}]^{+}$ & $\begin{array}{l}235.169 \\
217.1588 \\
199.1482^{b}\end{array}$ & Terpenoids & & \\
\hline 85 & 22.15 & $\mathrm{C}_{20} \mathrm{H}_{20} \mathrm{O}_{7}$ & Isosinensetin & $373.1283[\mathrm{M}+\mathrm{H}]^{+}$ & $\begin{array}{l}373.1281^{\mathrm{b}} \\
343.0812\end{array}$ & Flavonoids & $C P$ & [26] \\
\hline $86^{\mathrm{cd}}$ & 22.64 & $\mathrm{C}_{30} \mathrm{H}_{46} \mathrm{O}_{4}$ & $18 \beta$-Glycyrrhetinic acid & $471.3469[\mathrm{M}+\mathrm{H}]^{+}$ & $\begin{array}{l}417.3471^{b} \\
453.3362\end{array}$ & Terpenoids & $G R$ & [29] \\
\hline $87^{\mathrm{cd}}$ & 22.65 & $\mathrm{C}_{42} \mathrm{H}_{62} \mathrm{O}_{16}$ & Glycyrrhizic acid & $821.3926[\mathrm{M}-\mathrm{H}]^{-}$ & $\begin{array}{l}821.3915^{\mathrm{b}} \\
351.056 \\
113.0234\end{array}$ & Terpenoids & $G R$ & [20] \\
\hline 88 & 22.72 & $\mathrm{C}_{20} \mathrm{H}_{18} \mathrm{O}_{8}$ & Irisflorentin & $387.1073[\mathrm{M}+\mathrm{H}]^{+}$ & $\begin{array}{l}387.1073^{\mathrm{b}} \\
372.0843 \\
357.0603 \\
329.0654\end{array}$ & Flavonoids & $B H$ & [25] \\
\hline 89 & 22.84 & $\mathrm{C}_{20} \mathrm{H}_{20} \mathrm{O}_{7}$ & Sinensetin & $373.1282[\mathrm{M}+\mathrm{H}]^{+}$ & $\begin{array}{l}373.1281^{\mathrm{b}} \\
343.0809\end{array}$ & Flavonoids & $C P$ & [26] \\
\hline 90 & 22.98 & $\mathrm{C}_{18} \mathrm{H}_{14} \mathrm{O}_{8}$ & Dichotomitin & $359.0762[\mathrm{M}+\mathrm{H}]^{+}$ & $\begin{array}{l}359.0761^{\mathrm{b}} \\
344.0526 \\
326.0421 \\
299.0549\end{array}$ & Flavonoids & $B H$ & [24] \\
\hline $91^{\mathrm{a}}$ & 23.16 & $\mathrm{C}_{16} \mathrm{H}_{12} \mathrm{O}_{5}$ & Wogonin & $285.0757[\mathrm{M}+\mathrm{H}]^{+}$ & $\begin{array}{l}285.0756 \\
270.0521^{\mathrm{b}}\end{array}$ & Flavonoids & $S R$ & [19] \\
\hline 92 & 23.34 & $\mathrm{C}_{42} \mathrm{H}_{62} \mathrm{O}_{16}$ & isomer of Glycyrrhizic acid & $821.3922[\mathrm{M}-\mathrm{H}]^{-}$ & $\begin{array}{l}821.3919^{b} \\
351.0551 \\
113.0235\end{array}$ & Terpenoids & $G R$ & \\
\hline $93^{c}$ & 23.57 & $\mathrm{C}_{42} \mathrm{H}_{68} \mathrm{O}_{13}$ & Saikosaponin A & $825.4599[\mathrm{M}+\mathrm{COOH}]^{-}$ & $\begin{array}{l}779.4534^{\mathrm{b}} \\
617.40106 \\
59.0132\end{array}$ & Terpenoids & $B R$ & [26] \\
\hline 94 & 23.91 & $\mathrm{C}_{17} \mathrm{H}_{14} \mathrm{O}_{6}$ & Pectolinarigenin & $313.0703[\mathrm{M}-\mathrm{H}]^{-}$ & $\begin{array}{l}313.0701 \\
283.0233^{\mathrm{b}} \\
255.0286\end{array}$ & Flavonoids & $S R$ & [19] \\
\hline 95 & 23.99 & $\mathrm{C}_{21} \mathrm{H}_{22} \mathrm{O}_{8}$ & Nobiletin & $403.1388[\mathrm{M}+\mathrm{H}]^{+}$ & $\begin{array}{l}403.1388 \\
373.0917^{\mathrm{b}} \\
211.0238 \\
183.0288\end{array}$ & Flavonoids & $C P$ & [26] \\
\hline $96^{\mathrm{a}}$ & 24.04 & $\mathrm{C}_{19} \mathrm{H}_{18} \mathrm{O}_{6}$ & 6-Demethoxytangeretin & $343.1174[\mathrm{M}+\mathrm{H}]^{+}$ & $\begin{array}{l}343.1173 \\
313.0705^{\mathrm{b}} \\
285.0756\end{array}$ & Flavonoids & $C P$ & [26] \\
\hline $97^{\mathrm{c}}$ & 24.09 & $\mathrm{C}_{42} \mathrm{H}_{68} \mathrm{O}_{13}$ & Saikosaponin B1 & $825.4599[\mathrm{M}+\mathrm{COOH}]^{-}$ & $\begin{array}{l}779.4542^{b} \\
617.4028 \\
59.0132\end{array}$ & Terpenoids & $B R$ & \\
\hline $98^{\mathrm{a}}$ & 24.13 & $\mathrm{C}_{16} \mathrm{H}_{12} \mathrm{O}_{5}$ & Oroxylin A & $285.0758[\mathrm{M}+\mathrm{H}]^{+}$ & $\begin{array}{l}285.0757 \\
270.0523^{b} \\
168.0054\end{array}$ & Flavonoids & $S R$ & [19] \\
\hline
\end{tabular}


Table 1 (continued)

\begin{tabular}{|c|c|c|c|c|c|c|c|c|}
\hline No. & $\mathrm{RT}$ (min) & Formula & Potential compound & Detected $\mathrm{m} / \mathrm{z}$ & Characterized $\mathrm{MS}^{2}$ & Compound class & Herb & Refs \\
\hline 99 & 24.21 & $\mathrm{C}_{15} \mathrm{H}_{20} \mathrm{O}_{3}$ & Atractylenolide III & $249.1486[\mathrm{M}+\mathrm{H}]^{+}$ & $\begin{array}{l}231.1379^{b} \\
249.1481 \\
213.1276 \\
163.0752\end{array}$ & Terpenoids & $A M$ & {$[30]$} \\
\hline 100 & 24.49 & $\mathrm{C}_{22} \mathrm{H}_{24} \mathrm{O}_{9}$ & Heptamethoxyflavone & $433.1493[\mathrm{M}+\mathrm{H}]^{+}$ & $\begin{array}{l}403.1021 \\
433.1492^{b} \\
165.0546\end{array}$ & Flavonoids & $C P$ & [26] \\
\hline $101^{\mathrm{c}}$ & 24.7 & $\mathrm{C}_{42} \mathrm{H}_{68} \mathrm{O}_{13}$ & Saikosaponin D & $825.4594[\mathrm{M}+\mathrm{COOH}]^{-}$ & $\begin{array}{l}779.4537^{b} \\
617.4034 \\
59.0132\end{array}$ & Terpenoids & $B R$ & \\
\hline $102^{\mathrm{d}}$ & 25.67 & $\mathrm{C}_{20} \mathrm{H}_{20} \mathrm{O}_{7}$ & Tangeretin & $373.1280[\mathrm{M}+\mathrm{H}]^{+}$ & $\begin{array}{l}373.1278 \\
358.1043 \\
343.0808^{b} \\
328.0573\end{array}$ & Flavonoids & $C P$ & {$[26]$} \\
\hline 103 & 26.41 & $\mathrm{C}_{20} \mathrm{H}_{20} \mathrm{O}_{8}$ & 5-O-Demethylnobiletin & $389.1230[\mathrm{M}+\mathrm{H}]^{+}$ & $\begin{array}{l}389.1227^{b} \\
359.076 \\
341.0652\end{array}$ & Flavonoids & $C P$ & {$[21]$} \\
\hline 104 & 26.68 & $\mathrm{C}_{32} \mathrm{H}_{48} \mathrm{O}_{6}$ & Alisol C 23-acetate & $529.3526[\mathrm{M}+\mathrm{H}]^{+}$ & $\begin{array}{l}529.3521^{b} \\
469.3314 \\
451.3204 \\
415.2842\end{array}$ & Terpenoids & $A R$ & [31] \\
\hline 105 & 27.85 & $\mathrm{C}_{15} \mathrm{H}_{20} \mathrm{O}_{2}$ & Atractylenolide II & $233.1536[\mathrm{M}+\mathrm{H}]^{+}$ & $\begin{array}{l}233.1536^{\mathrm{b}} \\
215.1432 \\
187.1482 \\
151.0753\end{array}$ & Terpenoids & $A M$ & [30] \\
\hline 106 & 27.94 & $\mathrm{C}_{12} \mathrm{H}_{16} \mathrm{O}_{4}$ & Pogostone & $225.1122[\mathrm{M}+\mathrm{H}]^{+}$ & $\begin{array}{l}207.1015 \\
139.039 \\
81.0705^{\mathrm{b}}\end{array}$ & Miscellaneous & $P H$ & {$[32]$} \\
\hline 107 & 34.7 & $\mathrm{C}_{32} \mathrm{H}_{50} \mathrm{O}_{5}$ & Alisol B 23-acetate & $515.3733[\mathrm{M}+\mathrm{H}]^{+}$ & $\begin{array}{l}437.3412 \\
339.2679 \\
419.3305 \\
97.0653^{b}\end{array}$ & Terpenoids & $A R$ & {$[31]$} \\
\hline 108 & 35.79 & $\mathrm{C}_{18} \mathrm{H}_{30} \mathrm{O}_{2}$ & $\alpha$-Linolenic acid & $279.2318[\mathrm{M}+\mathrm{H}]^{+}$ & $\begin{array}{l}95.086 \\
81.0705 \\
67.055^{\mathrm{b}}\end{array}$ & Alkaloids & & \\
\hline
\end{tabular}

$R T$ retention time

${ }^{a}$ Representative retention time, as more than one peak was identified for this compound

${ }^{\mathrm{b}}$ Base fragment ion

${ }^{\mathrm{c}}$ Compounds detected using both the positive and negative electrospray ionization modes. $\mathrm{m} / \mathrm{z}$ : mass-to-charge ratio

${ }^{\mathrm{d}}$ Compounds identified by comparison with reference standards. Herb: Compound detected within herb experimentally and also the reference reported the source of the compound. Ref.: The references that reported the sources of the compounds. EH (Ephedrae Herba), GR (Glycyrrhizae Radix Et Rhizoma Praeparata Cum Melle), AS (Armeniacae Semen Amarum), CR (Cinnamomi Ramulus), PH (Pogostemonis Herba), AR (Alismatis Rhizoma), PP (Polyporus), AM (Atractylodis Macrocephalae Rhizoma), PR (Poria), BR (Bupleuri Radix), SR (Scutellariae Radix); PC (Pinelliae Rhizoma Praeparatum Cum Zingibere Et Alumine), ZR (Zingiberis Rhizoma Recens), AE (Asteris Radix Et Rhizoma), FF (Farfarae Flos), BH (Belamcandae Rhizoma), RE (Asari Radix Et Rhizoma), DR (Dioscoreae Rhizoma), AF (Aurantii Fructus Immaturus); CP (Citri Reticulatae Pericarpium)

$\left.-\mathrm{H}_{2} \mathrm{O}\right]^{+}$and $133.0887\left[\mathrm{M}+\mathrm{H}-\mathrm{H}_{2} \mathrm{O}-\mathrm{CH}_{3}\right]^{+}$. According to the literature [16], they are identified as L-ephedrine (19) and D-pseudoephedrine (20). The mass spectrum of compound 21 (methylephedrine) reveals a peak representing the protonated molecule $[\mathrm{M}+\mathrm{H}]^{+}$, at $\mathrm{m} / z$ 180.1382, and fragment ion peaks at $m / z 162.1276\left[\mathrm{M}+\mathrm{H}-\mathrm{H}_{2} \mathrm{O}\right]^{+}$and $147.1041\left[\mathrm{M}+\mathrm{H}-\mathrm{H}_{2} \mathrm{O}-\mathrm{CH}_{3}\right]^{+}$. The mass spectra of compounds 15 (L-norephedrine) and 16 (D-norpseudoephedrine) reveal the same peak at $\mathrm{m} / z 152.1069$, and $\mathrm{MS}^{2}$ peaks at $m / z$ 134.0965 $\left[\mathrm{M}+\mathrm{H}-\mathrm{H}_{2} \mathrm{O}\right]^{+}$and $117.0701\left[\mathrm{M}+\mathrm{H}-\mathrm{H}_{2} \mathrm{O}\right.$ $\left.-\mathrm{NH}_{3}\right]^{+}$. However, they exhibit different retention times. These compounds are phytochemicals present in Ephedrae Herba.

Compounds 3 (cytosine), 7 (nicotinic acid), and 9 (nicotinamide) were identified by comparing the retention times and $\mathrm{MS}^{2}$ fragmentation patterns with those of reference 
standards. Nicotinic acid and nicotinamide exhibit the same structural skeleton, and fragment ion peaks at $m / z 96.0448$ $[\mathrm{M}+\mathrm{H}-\mathrm{CO}]^{+}$are observed in the $\mathrm{MS}^{2}$ profiles. Their possible fragmentation pathways and library match results are shown in Fig. S4. The MS ${ }^{2}$ profile of compound 5 reveals a peak representing a protonated molecule, $[\mathrm{M}+\mathrm{H}]^{+}$, at $\mathrm{m} / \mathrm{z}$ 138.0550 and peaks at $\mathrm{m} / z, 110.0603[\mathrm{M}+\mathrm{H}-\mathrm{CO}]^{+}$and $94.0656[\mathrm{M}+\mathrm{H}-\mathrm{CO}-\mathrm{O}]^{+}$. Therefore, compound $\mathbf{5}$ is deduced to be trigonelline.

\section{Flavonoids}

Forty-nine compounds were identified as flavonoids. Compounds $\mathbf{4 7}$ and $\mathbf{5 1}$ were identified as narirutin and naringin, respectively, by comparison with the OTCML. Furthermore, compound $\mathbf{4 7}$ was confirmed using a reference standard. They were detected in both the positive and negative ESI modes, displaying similar MS and $\mathrm{MS}^{2}$ profiles that revealed peaks representing $[\mathrm{M}-\mathrm{H}]^{-}$ions at $m / z$ 579.1688. Fragment ions were represented by peaks at $\mathrm{m} / \mathrm{z} 271.0615$, owing to the loss of glucose (Glc) and rhamnose moieties [21]. Characterized fragment ions represented by peaks at $m / z, 151.0034$ and 119.0499 were generated by retro-Diels-Alder cleavage. Narirutin and naringin are flavonoid $O$-glycoside isomers distinguished by their different retention times. Compounds 87 (isosinensetin), 89 (sinensetin), 96 (6-demethoxytangeretin), 95 (nobiletin), and 102 (tangeretin) are polymethoxyflavones, bearing numerous methoxyl and/or hydroxyl groups on the basic structure. The mass spectra of these compounds show peaks representing $[\mathrm{M}+\mathrm{H}]^{+}$ions and characterized fragment ions due to continuous $\mathrm{CH}_{3}$ loss [26]. The $\mathrm{MS}^{2}$ profiles and library match results are shown in Fig. S5. As examples, the mass spectra of compounds $\mathbf{8 5}$ and $\mathbf{8 9}$ reveal peaks representing $[\mathrm{M}+\mathrm{H}]^{+}$ions at $m / z 373.1283$ and characterized fragment ions at $\mathrm{m} / z, 343.08[\mathrm{M}+\mathrm{H}$ $\left.-2 \mathrm{CH}_{3}\right]^{+}$. The spectra are very similar, and the compounds were identified using the OTCML by the different retention times and slight differences in the spectra. Compound 102 (tangeretin) was further confirmed using a reference standard. Compounds $\mathbf{3 0}$ and $\mathbf{3 3}$ showed similar $\mathrm{MS}^{2}$ patterns, but the molecular ions were different, indicating the same basic structure. These compounds were assigned as vicenin II [8] and isoschaftoside [20, 33], respectively. For example, the mass spectrum of compound 30 revealed peaks representing the $[\mathrm{M}-\mathrm{H}]^{-}$ion at $\mathrm{m} / \mathrm{z} 593.1482$ and fragment ions at $\mathrm{m} / \mathrm{z} 297.0750[\mathrm{M}$ $-\mathrm{H}-\mathrm{Glc}-\mathrm{Glc}^{-}, \mathrm{m} / \mathrm{z} 473.1062[\mathrm{M}-\mathrm{H}-120]^{-}, \mathrm{m} / \mathrm{z}$ $383.0753[\mathrm{M}-\mathrm{H}-210]^{-}$, and $m / z 353.0648[\mathrm{M}-\mathrm{H}$
$-240]^{-}$. These are characterized fragment ions of the hexose ring-opening reaction [33]. The similarities of the MS and $\mathrm{MS}^{2}$ profiles of compounds $\mathbf{3 7}, \mathbf{4 1}$, and $\mathbf{5 9}$ indicated isomers. By comparing the data in the OTCML combined with literature data [20], they were deduced as naringenin 7-O-(2- $\beta$-D-apiofuranosyl)- $\beta$-D-glucopyranoside (37), liquiritin apioside (41), and isoliquiritin apioside (59). The mass spectra of compounds $61,68,66$, and 70 revealed peaks representing $[\mathrm{M}-\mathrm{H}]^{-}$ions at $\mathrm{m} / z 445.07$ and dominant fragment ions at $m / z 269.04$, along with $[\mathrm{M}+\mathrm{H}]^{+}$ ions at $\mathrm{m} / \mathrm{z} 447.09$ and dominant fragment ions at $\mathrm{m} / \mathrm{z}$ 271.05. Individual herb pieces component mass spectra showed that these compounds, baicalin $(\mathbf{6 1})$, norwogonin7-glucuronide (68), norwogonin-8-glucuronide (66) and baicalein-6-glucuronide (70), were chemical components of Scutellariae Radix [19], and baicalin (61) was identified using a reference standard. Based on the literature [26], compounds $\mathbf{7 3}$ and $\mathbf{1 0 0}$ were assigned as hesperetin and heptamethoxyflavone, respectively. Compound 79 (isoliquiritigenin) was identified using a reference standard.

\section{Phenylpropanoids}

Fourteen compounds were identified as phenylpropanoids. Compounds 31 (1,3-dicaffeoylquinic acid), 46 (isochlorogenic acid B), 49 (3,5-dicaffeoylquinic acid) and $\mathbf{5 3}$ (isochlorogenic acid C) were identified using the OTCML. Compound 24 (chlorogenic acid) was identified using a reference standard. Compounds 31, 46, 49 and 53 were isomers with skeletons similar to those of quinic and caffeic acid, generating similar MS and $\mathrm{MS}^{2}$ profiles and distinguished by their retention times. For example, the $\mathrm{MS}^{2}$ profile of compound 46 revealed peaks representing fragment ions at $\mathrm{m} / \mathrm{z} 191.0547$ [quinic acid $-\mathrm{H}]^{-}, 179.0336$ [caffeic acid $-\mathrm{H}]^{-}$and 135.0440 [caffeic acid $\left.-\mathrm{CO}_{2}-\mathrm{H}\right]^{-}$. The mass spectrum of compound $\mathbf{3 8}$ (ferulic acid) showed peaks representing a $[\mathrm{M}-\mathrm{H}]^{-}$ion at $\mathrm{m} / \mathrm{z} 193.0492$ and the main fragment ions at $m / z 134.0362\left[\mathrm{M}-\mathrm{H}-\mathrm{CH}_{3}-\mathrm{CO}_{2}\right]^{-}$and $178.0258\left[\mathrm{M}-\mathrm{H}-\mathrm{CH}_{3}\right]^{-}$. Compounds 32 (p-coumaric acid), 57 (coumarin), 65 (bergaptol) and 25 (esculetin) were assigned using the OTCML.

\section{Phenolic Acids and Phenols}

Four phenolic acids were identified, and they exhibited the same fragmentation pattern. The $\mathrm{MS}^{2}$ profile of compound 12 (gallic acid) revealed peaks representing $[\mathrm{M}-\mathrm{H}]^{-}$at 
$\mathrm{m} / \mathrm{z} 169.0130$ and ions at $\mathrm{m} / \mathrm{z} 125.0233\left[\mathrm{M}-\mathrm{H}-\mathrm{CO}_{2}\right]^{-}$, $97.0285\left[\mathrm{M}-\mathrm{H}-\mathrm{CO}_{2}-\mathrm{CO}\right]^{-}$and $69.0337\left[\mathrm{M}-\mathrm{H}-\mathrm{CO}_{2}\right.$ $-\mathrm{CO}-\mathrm{CO}]^{-}$. The mass spectrum of compound 18 (protocatechuic acid) revealed a peak representing a base fragment ion at $m / z, 109.0284\left[\mathrm{M}-\mathrm{H}-\mathrm{CO}_{2}\right]^{-}$. Compound $\mathbf{4 4}$ (salicylic acid) was identified by comparison with a reference standard. All of these compounds exhibited successive losses of $\mathrm{H}_{2} \mathrm{O}, \mathrm{CO}$ and $\mathrm{CO}_{2}$ during fragmentation $[34,35]$.

Five phenols were identified. Compound $\mathbf{2 6}$ ( $p$-hydroxybenzaldehyde) produced several clear fragment ions at high collision energies. Compound 22 (protocatechualdehyde) was identified using the OTCML. The phenols also showed neutral losses of $\mathrm{CO}, \mathrm{CH}_{3}$ and $\mathrm{H}_{2} \mathrm{O}$ in the $\mathrm{MS}^{2}$ profiles.

\section{Terpenoids}

Thirteen terpenoids are identified. The mass spectra of compounds 82 and 87 reveal peaks representing $[\mathrm{M}+\mathrm{H}]^{+}$ions at $m / z 839.4061$ and 823.4108 , respectively. The mass spectrum of compound $\mathbf{8 2}$ (licorice-saponin G2) reveals peaks representing fragment ions at $\mathrm{m} / z$ 469.3314 [Aglycone $+\mathrm{H}$ $\left.-\mathrm{H}_{2} \mathrm{O}\right]^{+}$, 487.3412 [Aglycone $\left.+\mathrm{H}\right]^{+}$and 451.3212 [Aglycone $\left.+\mathrm{H}-2 \mathrm{H}_{2} \mathrm{O}\right]^{+}$[36]. Compound 87 displays a similar fragmentation pattern, yet is $16 \mathrm{Da}$ smaller than compound 82. Compound $\mathbf{8 7}$ was then confirmed as glycyrrhizic acid through a comparison between the negative ESI mode data, a reference standard, and literature data [20]. These spectra are shown in Fig.S6. The mass spectrum of compound 86, 18 $\beta$-glycyrrhetinic acid, reveals a peak representing $[\mathrm{M}+\mathrm{H}]^{+}$ at $m / z$ 471.3469. These are triterpenic acids. Compound $\mathbf{8 6}$ (18 $\beta$-glycyrrhetinic acid) was also identified using a reference standard.

The $\mathrm{MS}^{2}$ profile of compound $\mathbf{1 0 4}$ showed peaks representing a protonated molecule, $[\mathrm{M}+\mathrm{H}]^{+}$, at $\mathrm{m} / z 529.3526$ and dominant fragment ions at $\mathrm{m} / z 529.3521[\mathrm{M}+\mathrm{H}]^{+}$, $469.3314\left[\mathrm{M}+\mathrm{H}-\mathrm{HAc}^{+}, 451.3204[\mathrm{M}+\mathrm{H}-\mathrm{HAc}\right.$ $\left.-\mathrm{H}_{2} \mathrm{O}\right]^{+}$and $415.2842\left[\mathrm{M}+\mathrm{H}-\mathrm{C}_{4} \mathrm{H}_{8} \mathrm{O}-\mathrm{H}_{2} \mathrm{O}\right]^{+}$[31]. This compound was identified as alisol $\mathrm{C} 23$-acetate using the OTCML. The mass spectrum of compound 107, alisol B 23-acetate, revealed a peak representing $[\mathrm{M}+\mathrm{H}]^{+}$at $\mathrm{m} / \mathrm{z}$ 515.3733 .

The mass spectrum of compound 93 revealed peaks representing a $[\mathrm{M}+\mathrm{H}]^{+}$ion at $m / z, 781.4732$ and fragment ions at $m / z 455.3518\left[\mathrm{M}+\mathrm{H}-\mathrm{H}_{2} \mathrm{O}-\text { Fuc (fucose) Glc }\right]^{+}$ and $437.3412\left[\mathrm{M}+\mathrm{H}-2 \mathrm{H}_{2} \mathrm{O}-\mathrm{FucGlc}\right]^{+}$. This compound was identified as saikosaponin A by comparison with data obtained from the OTCML. The mass spectrum of compound 83 exhibited peaks representing $[\mathrm{M}+\mathrm{H}]^{+}$at $\mathrm{m} / \mathrm{z}$ 471.2016 and fragment ions at $m / z 425.1957[\mathrm{M}+\mathrm{H}-46]^{+}$ and 161.0597. According to the literature [23] and the data in the OTCML, it was limonin.

The mass spectra of compounds $\mathbf{9 9}$ and $\mathbf{1 0 5}$ revealed peaks representing $[\mathrm{M}+\mathrm{H}]^{+}$ions at $m / z 249.1486$ and
233.1536, respectively. They were identified as atractylenolide III and atractylenolide II, respectively, using the OTCML. The $\mathrm{MS}^{2}$ profile of atractylenolide III revealed peaks representing fragment ions at $\mathrm{m} / 2249.1481[\mathrm{M}+\mathrm{H}]^{+}$, $231.1379\left[\mathrm{M}+\mathrm{H}-\mathrm{H}_{2} \mathrm{O}\right]^{+}, 213.1276\left[\mathrm{M}+\mathrm{H}-2 \mathrm{H}_{2} \mathrm{O}\right]^{+}$and $203.1430\left[\mathrm{M}+\mathrm{H}-\mathrm{H}_{2} \mathrm{O}-\mathrm{CO}\right]^{+}[30]$.

\section{Other Phytochemicals}

Eleven compounds were identified by comparing the obtained data to the information in the OTCML, including the hydrophilic compounds 1 (sucrose), 2 (2-pyrrolidinecarboxylic acid), $\mathbf{6}$ (citric acid), $\mathbf{8}$ (uridine), 10 (adenosine) and $\mathbf{1 1}$ (guanosine). These compounds were also confirmed using reference standards.

\section{Quantification Analysis}

The extracted ion chromatograms (EICs) of 17 authentic standards compared with those of their corresponding detected compounds within QFPD granules are shown in Fig. 2. The HPLC-Q Exactive hybrid quadrupole-Orbitrap MS method was also used for quantification analysis of these 17 constituents within QFPD granules. The concentration of each constituent was obtained using the respective calibration curve and their contents within the QFPD granules are listed in Table 2.

\section{Compounds from Individual Herbs Within QFPD Granules}

In total, 265 compounds were putatively identified using the OTCML combined with manual verification from 20 herbs that are components of QFPD granules (Table S1), including 33 alkaloids, 106 flavonoids, 28 terpenoids, 41 phenylpropanoids, 10 phenolic acids, 18 phenols and 29 other phytochemicals. Of these, 163 compounds were from only one herb, and 102 compounds were from more than two herbs. Within the QFPD granules, 59 compounds were from only one herb and 49 compounds were from more than two herbs.

\section{Conclusions}

In this study, HPLC-Q Exactive hybrid quadrupole-Orbitrap MS coupled with the OTCML which is an automatic data analysis platform, was used to study the chemical profile of QFPD granules, an effective TCM prescribed to treat the symptoms of SARS-CoV-2 infections. Furthermore, manual 

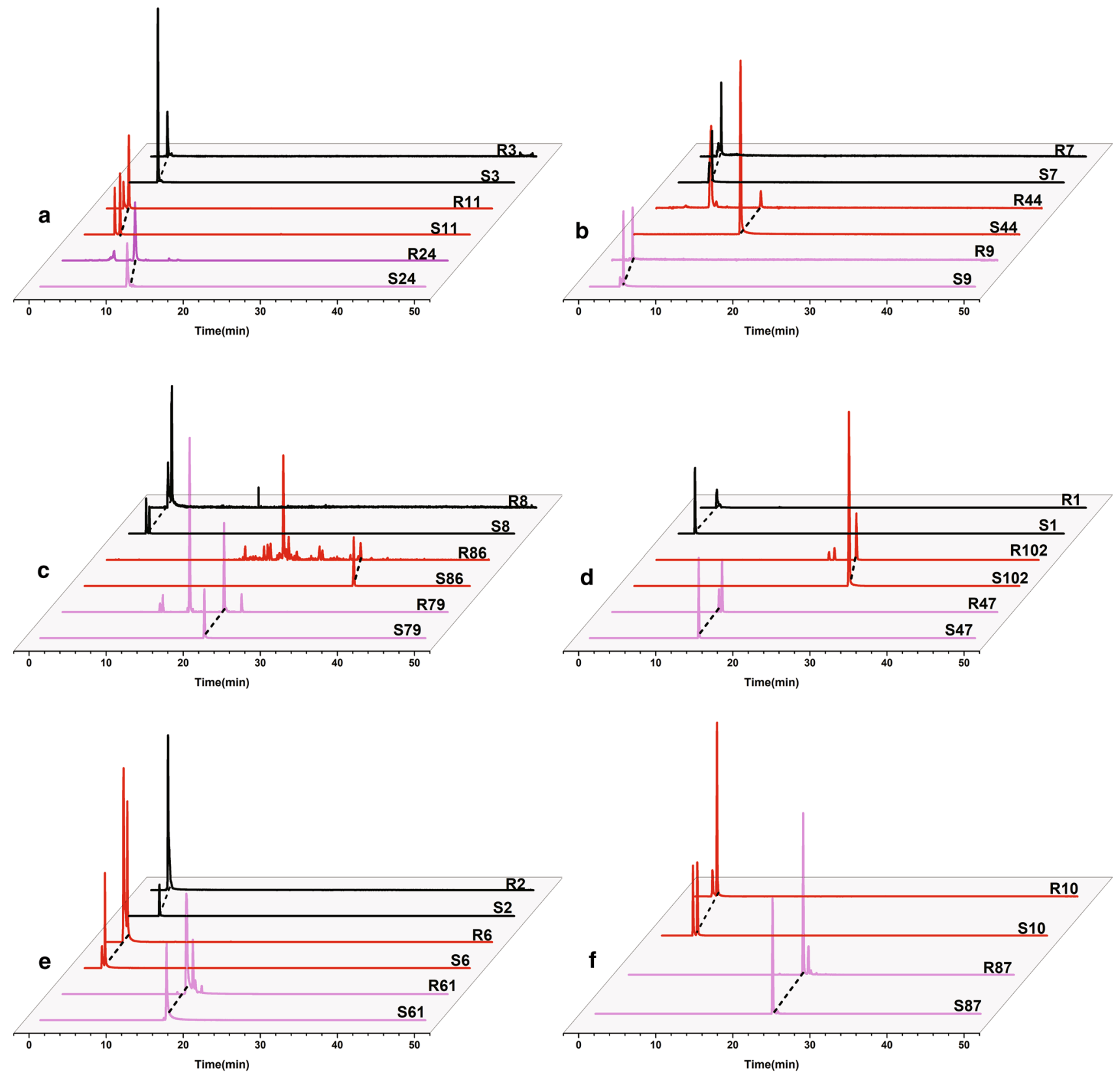

Fig. 2 Extracted ion chromatograms (EICs) of 17 authentic standards compared with those of the corresponding compounds detected within QFPD granules. A EICs of compounds 3, 11, and 24; B EICs of compounds 7, 44, and 9; C EICs of compounds 8, 86, and 79; D

verification ensured compound identification. A total of 108 Supplementary Information The online version contains supplementary material available at https://doi.org/10.1007/s10337-021-04085-0.

compounds were putatively identified from QFPD granules, including alkaloids, flavonoids, phenylpropanoids, phenolic acids, phenols, terpenoids and other phytochemicals. This allowed rapid chemical composition screening of QFPD granules, providing potentially valuable information for

EICs of compounds 1, 102, and 47; E EICs of compounds 2, 6, and 61; F EICs of compounds 10 and 87. $R$ sample from the QFPD granules, $S$ authentic standards

Acknowledgements This work was financially supported by the National Key R\&D Program of China (No. 2018YFA0900400), and the Non-profit Central Research Institute Fund of Chinese Academy of Medical Sciences (No.2020-PT320-004). 
Table 2 Contents of the constituents within QFPD granules

\begin{tabular}{llr}
\hline No & Compound & \multicolumn{1}{c}{ Conc. $(\mathrm{mg} / \mathrm{g})$} \\
\hline 1 & Sucrose & $0.683 \pm 0.185$ \\
2 & 2-Pyrrolidinecarboxylic acid & $0.803 \pm 0.017$ \\
3 & Cytosine & $0.009 \pm 0.0008$ \\
6 & Citric acid & $4.309 \pm 0.352$ \\
7 & Nicotinic acid & $0.011 \pm 0.0007$ \\
8 & Uridine & $0.029 \pm 0.005$ \\
9 & Nicotinamide & $0.006 \pm 0.0003$ \\
10 & Adenosine & $0.164 \pm 0.009$ \\
11 & Guanosine & $0.135 \pm 0.004$ \\
24 & Chlorogenic acid & $0.854 \pm 0.015$ \\
44 & Salicylic acid & $0.016 \pm 0.002$ \\
47 & Narirutin & $0.699 \pm 0.119$ \\
61 & Baicalin & $4.383 \pm 1.107$ \\
79 & Isoliquiritigenin & $0.007 \pm 0.00005$ \\
86 & 18 $\beta$-Glycyrrhetinic Acid & $0.0005 \pm 0.000007$ \\
87 & Glycyrrhizic acid & $2.199 \pm 0.127$ \\
102 & Tangeretin & $0.003 \pm 0.0003$ \\
\hline
\end{tabular}

Data present the (average \pm standard deviation) of three replicates. Conc. (mg/g): $\mathrm{mg}$ of the constituent/ $\mathrm{g}$ of QFPD granules

\section{Declarations}

Conflict of Interest The authors declare that there are no conflicts of interest.

Ethical Statement We certify that this manuscript is original, has not been previously published and will not be submitted elsewhere for publication while under consideration by Chromatographia. This study is not split into several parts and submitted to various journals. Results are presented clearly, honestly and without fabrication. No data, text, or theories by others are presented as our own.

Human and Animal Rights Explicit permission to submit has been received from all co-authors. All the authors whose name appear on the submission have contributed sufficiently to this study.

This manuscript does not contain any studies involving humans or animals.

\section{References}

1. Shi N, Liu B, Liang N, Ma Y, Ge Y, Yi H, Wo H, Gu H, Kuang Y, Tang S, Zhao Y, Tong L, Liu S, Zhao C, Chen R, Bai W, Fan Y, Shi Z, Li L, Liu J, Gu H, Zhi Y, Wang Z, Li Y, Li H, Wang J, Jiao L, Tian Y, Xiong Y, Huo R, Zhang X, Bai J, Chen H, Chen L, Feng Q, Guo T, Hou Y, Hu G, Hu X, Hu Y, Huang J, Huang Q, Huang S, Ji L, Jin H, Lei X, Li C, Wu G, Li J, Li M, Li Q, Li X, Liu H, Liu J, Liu Z, Ma Y, Mao Y, Mo L, Na H, Wang J, Song F, Sun S, Wang D, Wang M, Wang X, Wang Y, Wang Y, Wu W, Wu L, Xiao Y, Xie H, Xu H, Xu S, Xue R, Yang C, Yang K, Yang P, Yuan S, Zhang G, Zhang J, Zhang L, Zhao S, Zhao W, Zheng K, Zhou Y, Zhu J, Zhu T, Li G, Wang W, Zhang H,
Wang Y, Wang Y (2020) Pharmacol Res 161:105290. https:// doi.org/10.1016/j.phrs.2020.105290

2. Chen J, Wang YK, Gao Y, Hu LS, Yang JW, Wang JR, Sun WJ, Liang ZQ, Cao YM, Cao YB (2020) Biomed Pharmacother 129:110281. https://doi.org/10.1016/j.biopha.2020.110281

3. Zhao J, Tian S, Lu D, Yang J, Zeng H, Zhang F, Tu D, Ge G, Zheng Y, Shi T, Xu X, Zhao S, Yang Y, Zhang W (2020). Phytomedicine. https://doi.org/10.1016/j.phymed.2020.153315

4. National Medical Products Administration. https://www.nmpa. gov.cn/zhuanti/yqyjzxd/yqyjxd/20210302190503177.html.

5. Li Y, Shen Y, Yao CL, Guo DA (2020) J Pharm Biomed Anal 185:113215. https://doi.org/10.1016/j.jpba.2020.113215

6. Liu C, Guo DA, Liu L (2018) Phytomedicine 44:247-257. https://doi.org/10.1016/j.phymed.2018.03.006

7. Yang R, Liu H, Bai C, Wang Y, Zhang X, Guo R, Wu S, Wang J, Leung E, Chang H, Li P, Liu T, Wang Y (2020) Pharmacol Res 157:104820. https://doi.org/10.1016/j.phrs.2020.104820

8. Zhou YY, Gao WY, Gu XR, Chen ZQ, Zhao HY, Bian BL, Yang LX, Si N, Wang HJ, Tan Y (2020) China J Chin Materia Med 45:3035-3044. https://doi.org/10.19540/j.cnki.cjcmm.20200 423.202

9. Zhang F, Huang J, Liu W, Wang CR, Liu YF, Tu DZ, Liang XM, Yang L, Zhang WD, Chen HZ, Ge GB (2021) Food Chem Toxicol 149:111998. https://doi.org/10.1016/j.fct.2021.111998

10. Zhang Y, Cheng Y, Liu Z, Ding L, Qiu T, Chai L, Qiu F, Wang Z, Xiao W, Zhao L, Chen X (2017) J Chromatogr B 10611062:474-486. https://doi.org/10.1016/j.jchromb.2017.07.021

11. Chen MY, Liu W, Chou GX, Wang YL (2020) Acta Chin Med Pharmacol 48:62-66. https://doi.org/10.19664/j.cnki.10022392.200035

12. Zhang JY, Zhang XJ, Sun YK (2014) Chin J Inform TCM 21:71-73. https://doi.org/10.3969/j.issn.1005-5304.2014.05.022

13. Liu J, Xu YH, Zhang QQ, Zhu MH, Zhu ML, Zhou J (2020) Chin Tradit Patent Med 42:2003-2008. https://doi.org/10. 3969/j.issn.1001-1528.2020.08.008

14. Yang BY, Li M, Jing Y, Lai YY, Liu JL, Peng L (2018) Chin Tradit Herb Drugs 49:4349-4355. https://doi.org/10.7501/j.issn. 0253-2670.2018.18.020

15. Traditional Chinese Medicine Systems Pharmacology Database and Analysis Platform, Compilation prepared by Center for Bioinformatics, Northwest University.

16. Sun QH, Cao HJ, Zhou YY, Wang X, Jiang HQ, Gong LL, Yang Y, Rong R (2016) Biomed Chromatogr 30:1820-1834. https:// doi.org/10.1002/bmc.3758

17. Zheng L, Fang L, Cong H, Xiang T, Xue M, Yao Z, Wu B, Lin W (2015) Biomed Chromatogr 29:1750-1758. https://doi.org/ 10.1002/bmc.3489

18. Cheng XY, Zhang X, Liao M, Liang CJ, Diao XP, Zhang LT (2017) Chin Tradit Herbal Drugs 48:2390-2400. https://doi.org/ 10.7501/j.issn.0253-2670.2017.12.006

19. Qiao X, Li R, Song W, Miao WJ, Liu J, Chen HB, Guo DA, Ye M (2016) J Chromatogr A 1441:83-95. https://doi.org/10. 1016/j.chroma.2016.02.079

20. Xu T, Yang M, Li Y, Chen X, Wang Q, Deng W, Pang X, Yu K, Jiang B, Guan S, Guo DA (2013) Rapid Commun Mass Spectrom 27:2297-2309. https://doi.org/10.1002/rcm.6696

21. Zheng YY, Zeng X, Peng W, Wu Z, Su WW (2018) Phytochem Anal 30:278-291. https://doi.org/10.1002/pca.2812

22. Zhou J, Cai H, Tu S, Duan Y, Pei K, Xu Y, Liu J, Niu M, Zhang Y, Shen L, Zhou Q (2018) Molecules 23:3128. https://doi.org/ 10.3390/molecules23123128

23. Bai Y, Zheng Y, Pang W, Peng W, Wu H, Yao H, Li P, Deng W, Cheng J, Su W (2018) Molecules 23:803. https://doi.org/10. 3390/molecules23040803 
24. Zhang YY, Wang Q, Qi LW, Qin XY, Qin MJ (2011) J Pharm Biomed Anal 56:304-314. https://doi.org/10.1016/j.jpba.2011. 05.040

25. Li J, Li WZM, Huang W, Cheung AWH, Bi CWC, Duan R, Guo AJY, Dong TTX, Tsim KWK (2009) J Chromatogr A 1216:2071-2078. https://doi.org/10.1016/j.chroma.2008.05.082

26. Zheng GD, Zhou P, Yang H, Li YS, Li P, Liu EH (2013) Food Chem 136:604-611. https://doi.org/10.1016/j.foodchem.2012. 08.040

27. Cheng M, Ding L, Kan H, Zhang H, Jiang B, Sun Y, Cao S, Li W, Koike K, Qiu F (2019) J Nat Med 73:847-854. https://doi. org/10.1007/s11418-019-01329-0

28. Wang CC, Liu YY, Yang HT, Zhang QY, Liao M, Zhang X, Zhang LT (2016) Chin Tradit Herbal Drugs 47:2534-2539. https://doi.org/10.7501/j.issn.0253-2670.2016.14.024

29. Xu L, Liu B, Wang F, Gao XH, Wang YQ, Wang HJ, Li N, Zhang JY (2018) China J Chin Materia Med 43:4534-4540. https://doi.org/10.19540/j.cnki.cjcmm.2018.0120

30. Sun X, Wen H-M, Cui XB, Lu TL, Li W, Shan CX (2016) Chin Tradit Herbal Drugs 47:3494-3501. https://doi.org/10.7501/j. issn.0253-2670.2016.19.023

31. Zhao W, Huang X, Li X, Zhang F, Chen S, Ye M, Huang M, Xu W, Wu S (2015) Molecules 20:13958-13981. https://doi.org/10. 3390/molecules200813958
32. Li K, Zhang H, Xie H, Liang Y, Wang X, Ito Y (2011) J Liq Chromatogr Relat Technol 34:1617-1629. https://doi.org/10. 1080/10826076.2011.580486

33. Zhang K, Xu X, Li T, Song YL, Zhao YF, Song QQ, Tu PF (2020) China J Chin Materia Med 45:899-909. https://doi.org/ 10.19540/j.cnki.cjcmm.20191106.201

34. Huang WP, Tan T, Li ZF, OuYang H, Xu X, Zhou B, Feng YL (2018) J Pharm Biomed Anal 154:236-244. https://doi.org/10. 1016/j.jpba.2018.02.020

35. Shen Y, Feng Z, Yang M, Zhou Z, Han S, Hou J, Li Z, Wu W, Guo DA (2018) J Sep Sci 41:1888-1895. https://doi.org/10.1002/jssc. 201701134

36. Zheng ZG, Xu YH, Liu F, Zhao TT, Wang RX, Huang PY, Wang RS, Yang AP, Zhu Q (2019) J Pharm Biomed Anal 169:127-132. https://doi.org/10.1016/j.jpba.2019.03.007

Publisher's Note Springer Nature remains neutral with regard to jurisdictional claims in published maps and institutional affiliations. 